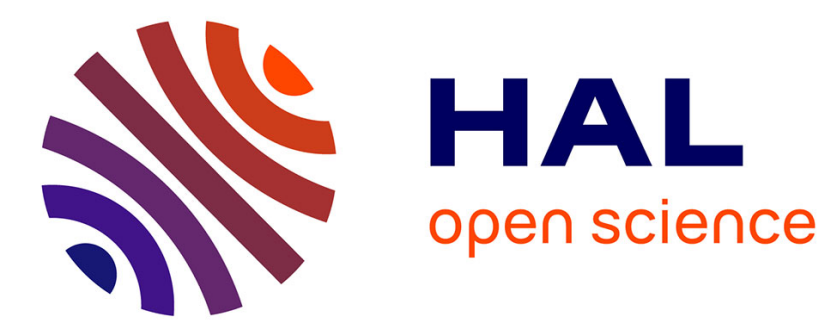

\title{
Modular Aspects of Rewrite-Based Specifications
}

Bernhard Gramlich

\section{To cite this version:}

Bernhard Gramlich. Modular Aspects of Rewrite-Based Specifications. [Research Report] RR-3330,

INRIA. 1997, pp.20. inria-00073359

\section{HAL Id: inria-00073359 \\ https://hal.inria.fr/inria-00073359}

Submitted on 24 May 2006

HAL is a multi-disciplinary open access archive for the deposit and dissemination of scientific research documents, whether they are published or not. The documents may come from teaching and research institutions in France or abroad, or from public or private research centers.
L'archive ouverte pluridisciplinaire HAL, est destinée au dépôt et à la diffusion de documents scientifiques de niveau recherche, publiés ou non, émanant des établissements d'enseignement et de recherche français ou étrangers, des laboratoires publics ou privés. 
INSTITUT NATIONAL DE RECHERCHE EN INFORMATIQUE ET EN AUTOMATIQUE

\section{Modular Aspects of Rewrite-Based Specifications}

Bernhard Gramlich

\section{$\mathbf{N}^{\circ} 3330$}

Décembre 1997

THÈME 2 



\title{
RINRIA
}

\section{Modular Aspects of Rewrite-Based Specifications}

\author{
Bernhard Gramlich* \\ Thème 2 - Génie logiciel \\ et calcul symbolique \\ Projet PROTHEO
}

Rapport de recherche $\mathrm{n}^{\circ} 3330$ - Décembre 1997 - 20 pages

\begin{abstract}
We investigate modular properties of term rewriting systems, the basic operational formalism for equational specifications. First we study sufficient conditions for the preservation of the termination property under disjoint (and more general) combinations of term rewriting systems. By means of a refined analysis of existing approaches we show how to prove several new asymmetric preservation results. For this purpose we introduce two interesting new properties of term rewriting systems related to collapsing reductions: uniquely collapsing and collapsing confluent. We discuss these properties w.r.t. well-known confluence, consistency and normal form properties, and show that they are modular for left-linear systems, but not in general.
\end{abstract}

Key-words: Equational specification, term rewriting, modularity, termination, confluence, collapsing reduction.

(Résumé : tsvp)

This work was supported by a Marie Curie Research Fellowship of the European Community under contract No. ERBFMBICT961235.

An abridged version of this paper will appear in the final LNCS-proceedings of the 12th Int. Workshop on Algebraic Development Techniques (WADT'97), Tarquinia, Italy, June 1997.

* e-mail: gramlich@loria.fr, url: http://ннн.loria.fr/ gramlich

Unité de recherche INRIA Lorraine

Technopôle de Nancy-Brabois, Campus scientifique,

615 rue de Jardin Botanique, BP 101, 54600 VILLERS LÈS NANCY (France)

Téléphone : (33) 83593030 - Télécopie : (33) 83278319

Antenne de Metz, technopôle de Metz 2000, 4 rue Marconi, 55070 METZ

Téléphone : (33) 87203500 - Télécopie : (33) 87763977 


\section{Aspects modulaires de spécifications basées sur la réécriture}

Résumé : Nous étudions des propriétés modulaires de systèmes de réécriture qui constituent le formalisme opérationnel de base des spécifications équationnelles. Dans un premier temps nous considérons des conditions suffisantes pour la préservation de la terminaison de systèmes de réécriture sous des combinaisons disjointes (et plus générales). À partir d'une analyse détaillée d'approches existantes nous montrons plusieurs nouveaux resultats asymétriques de préservation. Dans ce contexte nous introduisons deux nouvelles propriétés intéressantes de réductions effondrantes. Nous les comparons avec d'autres propriétés bien connues de confluence, de consistance et de formes normales, et nous montrons qu'elles sont modulaires pour les systèmes linéaires à gauche, mais pas pour des systèmes arbitraires.

Mots-clé : Spécification équationnelle, réécriture, modularité, terminaison, confluence, réduction effondrante. 


\section{Introduction}

The study of the modularity behaviour of rewrite systems (w.r.t. to the preservation of important properties) under various types of combinations has become a very active and fruitful area of research (cf. [15], [18], [9] for surveys). This field is of utmost importance for the modular construction of (the operational version of) equational specifications with desirable properties as well as for their structured analysis by a divide-and-conquer approach.

Here we shall be concerned with the question under which conditions disjoint unions of rewrite systems inherit termination from their constituent systems. In particular, we are interested in asymmetric preservation conditions which have only partially been explored up to now. This analysis naturally leads to a thorough investigation of collapsing reductions and the role of (non-)left-linearity partially refining the existing analyses of [25] and $[13,14,22]$. Besides the implications for the preservation of termination under (disjoint) combinations this analysis of collapsing reduction and (non-)left-linearity may have other potential applications, too.

The rest of the paper is organized as follows. In Section 2 we recall some basic terminology about term rewriting and modularity. In Section 3 we give a brief survey of the basic approaches and (some) modularity results for termination and prepare our analysis. The main results of the paper are presented in Sections 4,5 and 6. Finally we briefly discuss possible extensions (of the results on preservation of termination) for non-disjoint combinations of systems.

\section{Preliminaries}

We assume familiarity with the basic no(ta)tions, terminology and theory of term rewriting (cf. e.g. [4], [10]) but recall some no(ta)tions for the sake of readability. The set of terms over some given signature $\mathcal{F}$ and some (disjoint) countably infinite set $\mathcal{V}$ of variables is denoted by $\mathcal{T}(\mathcal{F}, \mathcal{V})$. Variables are denoted by $x, y, z, \ldots$ Positions (in terms) are ordered by the prefix ordering $\leq$ as usual. Concatenation of positions is denoted by juxtaposition. The 'empty' root position is denoted by $\lambda$. Two positions $p$ and $q$ are said to be parallel (or independent, disjoint) if neither $p \leq q$ nor $q \leq p$. The set of positions of a term $s$ is denoted by $\operatorname{Pos}(s)$. The sets of variable positions and of non-variable, i.e., function symbol, positions of $s$ are denoted by $\mathcal{V} \operatorname{Pos}(s)$ and $\mathcal{F} \operatorname{Pos}(s)$, respectively. The subterm of $s$ at some position $p \in \operatorname{Pos}(s)$ is denoted by $s / p$.

A term rewriting system $(\mathrm{TRS})$ is a pair $(\mathcal{F}, \mathcal{R})$ consisting of a signature $\mathcal{F}$ and a set $\mathcal{R}$ of rewrite rules over $\mathcal{F}$, i.e., pairs $(l, r)$ - also denoted by $l \rightarrow r$ - with $l, r \in \mathcal{T}(\mathcal{F}, \mathcal{V})$. Here we require that $l$ is not a variable, and that all variables of $r$ occur in $l$ (this excludes only degenerate cases). Instead of $(\mathcal{F}, \mathcal{R})$ we also write $\mathcal{R}^{\mathcal{F}}$ or simply $\mathcal{R}$ if $\mathcal{F}$ is clear from the context or irrelevant. For reduction steps with the rewrite relation $\rightarrow_{\mathcal{R}}=\rightarrow$ induced by $\mathcal{R}$ we sometimes add additional information as in $s \rightarrow_{p, \sigma, l \rightarrow r} t$ with the obvious meaning. Furthermore, we make free use of context notations like $s=C[\sigma(l)] \rightarrow_{l \rightarrow r} C[\sigma(r)]=t$ or $s=C\left[s_{1}, \ldots, s_{n}\right]_{p_{1}, \ldots, p_{n}}$ where the $p_{i}$ 's indicate the respective positions of the $s_{i}$ 's. If in a

$\operatorname{RR} \mathbf{n}^{\circ} 3330$ 
reduction sequence (or derivation) $D: s_{0} \rightarrow^{*} s_{n}$ every step is uniquely specified, e.g., by indicating the position $p$ of the redex contracted, the applied rule $l \rightarrow r$ (and redundantly the matching substitution $\sigma$ ), then we speak of a labelled derivation. The innermost reduction relation $\longrightarrow$ (induced by $\mathcal{R}$ ) is given by: $s \underset{i}{\rightarrow} t$ if $s=C[\sigma(l)] \rightarrow_{l \rightarrow r} C[\sigma(r)]=t$ for some $l \rightarrow r \in \mathcal{R}$, some context $C[$.$] and some substitution \sigma$ such that no proper subterm of $\sigma(l)$ is reducible. A TRS is non-overlapping if it has no critical pairs (note that an overlap of a rule with itself at root position is not considered to be critical). It is an overlay or overlaying system if critical overlaps between rules of $\mathcal{R}$ occur only at the root position. An inside critical pair is obtained by overlapping some rule into another one properly below the root. A TRS $\mathcal{R}$ is terminating or strongly normalizing $(\mathrm{SN})$ if $\rightarrow_{\mathcal{R}}$ is terminating, i.e., if there is no infinite derivation $s_{0} \rightarrow_{\mathcal{R}} s_{1} \rightarrow_{\mathcal{R}} s_{2} \ldots \mathcal{R}$ is innermost terminating if $\underset{i}{\longrightarrow}$ is terminating. It is confluent or Church-Rosser (CR) if ${ }^{*} \leftarrow \circ \rightarrow^{*} \subseteq \rightarrow^{*} \circ^{*} \leftarrow$ or equivalently $\leftrightarrow^{*} \subseteq \rightarrow^{*} 0^{*} \leftarrow$. Confluence plus termination is also called completeness or convergence. $\mathcal{R}$ is locally confluent or weakly Church-Rosser (WCR) if $\leftarrow \circ \rightarrow \subseteq \rightarrow^{*} \circ * \leftarrow$. The set of irreducible terms (or terms in normal form) is denoted by $\operatorname{NF}(\mathcal{R})$. $\mathcal{R}$ has unique normal forms (UN) if for all terms $s, t, s \leftrightarrow^{*} t$ and $s, t \in \mathrm{NF}(\mathcal{R})$ imply $s=t$. $\mathcal{R}$ has unique normal forms w.r.t. reduction $\left(\mathrm{UN}^{\rightarrow}\right)$ if for all $s, t, u, s^{*} \leftarrow u \rightarrow^{*} t$ and $s, t \in \mathrm{NF}(\mathcal{R})$ imply $s=t$. $\mathcal{R}$ has the normal form property (NF) if for all $s, t, s \leftrightarrow^{*} t$ and $t \in \operatorname{NF}(\mathcal{R})$ imply $s \rightarrow^{*} t$. $\mathcal{R}$ is consistent (CON) if $x \leftrightarrow^{*} y$ implies $x=y$, and consistent w.r.t. reduction $\left(\mathrm{CON}^{\rightarrow}\right)$ if $x^{*} \leftarrow s \rightarrow^{*} y$ implies $x=y$. Local versions of certain properties like termination and confluence (for instance: $t$ is terminating) also make sense, with the obvious interpretation. A rewrite rule $l \rightarrow r$ is collapsing if $r$ is a variable. A TRS is non-collapsing if it contains no collapsing rule. It is left-linear if any variable occurs at most once in any left hand side.

Next we recall some basic terminology for analyzing rewriting in disjoint unions (cf. [24], [15]). A property $P$ of rewrite systems is said to be modular (for disjoint systems) if, for all disjoint systems $\mathcal{R}_{b}{ }^{\mathcal{F}_{b}}, \mathcal{R}_{w}{ }^{\mathcal{F}_{w}}$ and $\mathcal{R}^{\mathcal{F}}$ with $\mathcal{R}^{\mathcal{F}}=\left(\mathcal{R}_{b} \uplus \mathcal{R}_{w}\right)^{\mathcal{F}_{b} \uplus \mathcal{F}_{w}}: P\left(\mathcal{R}_{b}{ }^{\mathcal{F}_{b}}\right) \wedge$ $P\left(\mathcal{R}_{w}{ }^{\mathcal{F}_{w}}\right) \Longleftrightarrow P\left(\mathcal{R}^{\mathcal{F}}\right)$. We say that $P$ is modular for (disjoint unions of) TRSs satisfying $Q$ if $(P \wedge Q)$ is modular. Let us assume subsequently that $\mathcal{R}_{b}{ }^{\mathcal{F}_{b}}$ and $\mathcal{R}_{w}{ }^{\mathcal{F}_{w}}$ are disjoint TRSs with $\mathcal{R}^{\mathcal{F}}$ (or $\mathcal{R}=\mathcal{R}_{b} \oplus \mathcal{R}_{w}$ ) denoting their disjoint union. Furthermore we shall use the abbreviating notations $\mathcal{T}=\mathcal{T}(\mathcal{F}, \mathcal{V})$ and $\mathcal{T}_{i}=\mathcal{T}\left(\mathcal{F}_{i}, \mathcal{V}\right)$ for $i=b, w$. First of all, in order to achieve better readability we introduce the mostly used chromatic terminology. Many definitions, notations and case distinctions are symmetric w.r.t. the two systems. The non-explicit case is therefore often indicated in parentheses (or omitted). Function symbols from $\mathcal{F}_{b}\left(\mathcal{F}_{w}\right)$ are called black (white). Variables are transparent, i.e., have no colour. A term $s \in \mathcal{T}$ is called black (white) if $s \in \mathcal{T}_{b}\left(s \in \mathcal{T}_{w}\right)$. We say that $s$ is top black (top white, top transparent) if $\operatorname{root}(s) \in \mathcal{F}_{b}\left(\operatorname{root}(s) \in \mathcal{F}_{w}\right.$, $\left.\operatorname{root}(s) \in \mathcal{V}\right){ }^{1}$ Terms in $\mathcal{T}_{b} \cup \mathcal{T}_{w}$ are called homogeneous, terms from $\mathcal{T} \backslash\left(\mathcal{T}_{b} \cup \mathcal{T}_{w}\right)$ mixed. If $s=C\left[s_{1}, \ldots, s_{n}\right]$ is top black (top white), and the $s_{i}$ 's are the maximal top white (top black) subterms in $s$, we also write $s=C^{b} \llbracket s_{1}, \ldots, s_{n} \rrbracket\left(s=C^{w} \llbracket s_{1}, \ldots, s_{n} \rrbracket\right)$. In this case the $s_{i}$ 's are the

\footnotetext{
${ }^{1}$ Note that a top transparent term must be a variable. Hence, this case is degenerate here for disjoint unions. However, for constructor sharing and composable systems, this terminology can be conveniently extended.
} 
white (black) principal subterms or aliens of $s$. We use $s=C \llbracket s_{1}, \ldots, s_{n} \rrbracket$ to denote either of the cases and say that the $s_{i}$ 's are the principal subterms (or aliens) of $s$. The rank of $s \in \mathcal{T}$ is defined by: $\operatorname{rank}(s)=0$ if $x \in \mathcal{V}, \operatorname{rank}(s)=1$ if $s \in\left(\mathcal{T}_{b} \cup \mathcal{T}_{w}\right) \backslash \mathcal{V}$, and $\operatorname{rank}(s)=1+\max \left\{\operatorname{rank}\left(s_{i}\right) \mid 1 \leq i \leq n\right\}$ if $s=C \llbracket s_{1}, \ldots, s_{n} \rrbracket(n \geq 1)$. The rank of a derivation $D: s_{1} \rightarrow s_{2} \rightarrow \ldots$ is $\min \left\{\operatorname{rank}\left(s_{i}\right) \mid s_{i}\right.$ occurs in $\left.D\right\}$. For $s \rightarrow t$, if $s$ reduces to $t$ by applying some rule in one of the principal subterms of $s$, we write $s \stackrel{i}{\rightarrow} t$, otherwise $s \stackrel{0}{\rightarrow} t$.

The relations $\stackrel{i}{\rightarrow}$ and $\stackrel{\circ}{\rightarrow}$ are called inner and outer reduction, respectively. A rewrite step $s \rightarrow t$ is destructive at level 1 if the root symbols of $s$ and $t$ have different colours, i.e., if either $s$ is top black and $t$ top white or top transparent (i.e., a variable), or $s$ is top white and $t$ top black or top transparent. The rewrite step $s \rightarrow t$ is destructive at level $n+1$ if $s=C \llbracket s_{1}, \ldots, s_{j}, \ldots, s_{n} \rrbracket \stackrel{i}{\rightarrow} C\left[s_{1}, \ldots, t_{j}, \ldots, s_{n}\right]$ with $s_{j} \rightarrow t_{j}$ destructive at level $n$. A step $s \rightarrow t$ is destructive if it is destructive at some level $n \geq 1$. Note that if a rewrite step is destructive then the applied rule must be collapsing. A term $s \in \mathcal{T}$ is called preserved if no derivation issuing from $s$ contains a destructive step. We say that $s$ is inner preserved if all its principal subterms are preserved.

Due to lack of space we omit numerous basic facts about rewriting in disjoint unions which we shall tacitly use in the sequel (cf. e.g. [24], [15]).

\section{Some Known Results}

It is well-known that confluence is modular but termination is not ([24]):

Example 1 ([24]) The disjoint TRSs

$$
\mathcal{R}_{b}=\{f(a, b, x) \rightarrow f(x, x, x)\} \quad \mathcal{R}_{w}=\left\{\begin{array}{l}
G(x, y) \rightarrow x \\
G(x, y) \rightarrow y
\end{array}\right\}
$$

are terminating, but $\mathcal{R}=\mathcal{R}_{b} \oplus \mathcal{R}_{w}$ is not, due to the cyclic derivation $f(a, b, G(a, b)) \rightarrow_{\mathcal{R}_{b}} f(G(a, b), G(a, b), G(a, b)) \rightarrow_{\mathcal{R}_{w}}^{+} f(a, b, G(a, b)) \rightarrow \ldots$

The non-confluence of the second system above is not essential for the existence of such counterexamples, since even completeness is not modular ([23]). A simple counterexample is the following.

Example 2 ([5]) The disjoint TRSs

$$
\mathcal{R}_{b}=\left\{\begin{aligned}
f(a, b, x) & \rightarrow f(x, x, x) \\
a & \rightarrow c \\
b & \rightarrow c \\
f(x, y, z) & \rightarrow c
\end{aligned}\right\} \quad \mathcal{R}_{w}=\left\{\begin{aligned}
& K(x, y, y) \rightarrow x \\
& K(y, y, x) \rightarrow x
\end{aligned}\right\}
$$

$\operatorname{RR} \mathbf{n}^{\circ} 3330$ 
are terminating and confluent hence complete, but again their disjoint union $\mathcal{R}=\mathcal{R}_{b} \oplus \mathcal{R}_{w}$ allows a cycle:

$$
f(a, b, K(a, c, b)) \rightarrow_{\mathcal{R}_{b}} f\left(K(a, c, b)^{3}\right) \rightarrow_{\mathcal{R}}^{+} f(a, b, K(a, c, b)) .
$$

The known positive results for modularity of termination are, roughly speaking, based on three different approaches concerning the essential ideas and proof structures (cf. [9]):

(1) a general approach via an abstract structure theorem where the basic idea is to reduce non-termination in the union to non-termination of a slightly extended generic version of one of the systems ([7], [19]),

(2) a modular approach via modularity of innermost termination where sufficient criteria for the equivalence of innermost termination and general termination are combined with the modularity of innermost termination ([8]), and

(3) a syntactic approach via left-linearity which in essence is based on commutation and uniqueness properties of (collapsing) reduction in left-linear systems ([25], [14, 22]).

Due to lack of space we cannot give a more detailed account of the (numerous) papers on the subject and the above classification. We only mention some results to which we refer later on. Virtually all proofs for showing preservation results for termination under disjoint unions (and more general combinations) rely on properties of minimal counterexamples of the following form: If the union of two disjoint terminating systems $\mathcal{R}_{b}$ and $\mathcal{R}_{w}$ (having some properties) is non-terminating, then a minimal counterexample in the union must enjoy certain properties and, consequently, $\mathcal{R}_{b}$ and $\mathcal{R}_{w}$ must satisfy certain (additional) properties. Since in general the role of $\mathcal{R}_{b}$ and $\mathcal{R}_{w}$ in minimal counterexamples need not be symmetric this may naturally entail corresponding (positive) symmetric and asymmetric preservation results. In the literature this observation has been systematically exploited for approach (1) above, and partially also for (3). Here we shall show how to do this for (2) and how to considerably refine the existing analysis for (3). In all obtained new results (on the preservation of termination) one of the systems must be non-collapsing but not necessarily the other one.

The main (symmetric) preservation result corresponding to (2) above is the following (here and subsequently we focus on the non-trivial implication of the corresponding modularity result).

Theorem 1 ([8]) If $\mathcal{R}_{b}, \quad \mathcal{R}_{w}$ are terminating, confluent and overlaying then $\mathcal{R}_{b} \oplus \mathcal{R}_{w}$ is terminating (as well as confluent and overlaying).

Two symmetric results and an asymmetric one corresponding to approach (3) above are the following.

Theorem 2 ([25]) If $\mathcal{R}_{b}, \mathcal{R}_{w}$ are terminating, confluent and left-linear, then $\mathcal{R}_{b} \oplus \mathcal{R}_{w}$ is terminating (as well as confluent and left-linear). 
Theorem $3([\mathbf{2 2}, \mathbf{1 4}])$ If $\mathcal{R}_{b}, \mathcal{R}_{w}$ are terminating, consistent w.r.t. reduction and leftlinear, then $\mathcal{R}_{b} \oplus \mathcal{R}_{w}$ is terminating (as well as consistent w.r.t. reduction and left-linear).

Theorem 4 ([25]) Let $\mathcal{R}_{b}, \mathcal{R}_{w}$ be terminating TRSs. If $\mathcal{R}_{b}$ is non-collapsing and $\mathcal{R}_{w}$ is left-linear and confluent then $\mathcal{R}_{b} \oplus \mathcal{R}_{w}$ is terminating.

\section{An Asymmetric Version of the Modular Approach}

We shall show that in Theorem 1, if one of the two terminating systems is non-collapsing, confluent and overlaying, then the other system need only be confluent but not overlaying for ensuring termination of $\mathcal{R}_{b} \oplus \mathcal{R}_{w}$.

First we introduce some definitions for locally confluent TRSs. In such systems a term is terminating if and only if it is complete (by Newman's Lemma). Hence we can define $\Phi(t)=C\left[t_{1} \downarrow, \ldots, t_{n} \downarrow\right]$ for $t=C\left[t_{1}, \ldots, t_{n}\right]$ such that $t_{1}, \ldots, t_{n}$ are the (uniquely defined) maximal complete subterms of $t$. Here $t_{i} \downarrow$ denotes the unique normal form of $t_{i}$. Clearly we have $t \rightarrow^{*} \Phi(t)$.

If $s \rightarrow t$ by contracting a terminating redex in $s$ (i.e., $s \rightarrow_{p} t$ for some $p \in \operatorname{Pos}(s)$, with $s / p$ terminating), we write $s \rightarrow_{\mathrm{sn}} t .^{2}$ If $s \rightarrow t$ by contracting a non-terminating redex in $s$ (i.e., $s \rightarrow_{p} t$ for some $p \in \operatorname{Pos}(s)$, with $s / p$ non-terminating), we write $s \rightarrow_{\neg s n} t$. Clearly, every reduction step can be written as $s \rightarrow_{\mathrm{sn}} t$ or $s \rightarrow_{\neg \text { sn }} t$. We observe that whenever $s \rightarrow_{\mathrm{sn}} t$ by a root reduction step then this implies that $s$ is terminating. The relation $\rightarrow_{\mathrm{sn}}$ is terminating for any TRS (which is easily proved by structural induction). Furthermore, every infinite derivation contains infinitely many $\rightarrow_{\neg \text { sn }}$-steps (which are not $\rightarrow_{\mathrm{sn}}$-steps). Contracting a terminating redex is compatible with the transformation $\Phi$, in the following sense.

Lemma 5 ([8]) Let $\mathcal{R}$ be locally confluent. If $s \rightarrow_{\mathrm{sn}} t$ then $\Phi(s) \rightarrow^{*} \Phi(t)$.

Lemma 6 Suppose $\mathcal{R}$ is a locally confluent TRS. Let $l \rightarrow r$ be a rule of $\mathcal{R}$ such that there exists no inside critical peak by overlapping some other (or the same) rule of $\mathcal{R}$ into $l \rightarrow r$ properly below the root. Let $\sigma$ be a substitution such that $\sigma l$ is not complete. Then $\Phi(\sigma l)=(\Phi \circ \sigma) l$ (where $\Phi \circ \sigma$ is the substitution defined by $(\Phi \circ \sigma) x=\Phi(\sigma x)$ ). In particular, if additionally $\sigma x$ is complete for all $x \in \operatorname{Var}(l)$, then $\Phi(\sigma l)=(\Phi \circ \sigma) l=(\sigma \downarrow) l$ and all proper subterms of $\Phi(\sigma l)$ are irreducible. ${ }^{3}$

Proof: We have to show that normalization of all maximal complete subterms in $\sigma l$ can be achieved by normalizing all maximal complete subterms in the "substitution part $\sigma$ of $\sigma l$ ". If no subterm of $\sigma l$ is complete we clearly obtain $\Phi(\sigma l)=(\Phi \circ \sigma) l=\sigma l$ by definition of $\Phi$. Hence, we may assume that some subterm of $\sigma l$ is complete. Let $\sigma l=C\left[t_{1}, \ldots, t_{n}\right]$, $n \geq 1$, where the $t_{i}$ 's are the maximal complete subterms of $\sigma l$, let's say with $\sigma l / p_{i}=t_{i}$. Note that, due to the assumption that $\sigma l$ is not complete, we have $\lambda<p_{i}$ for all $p_{i}$. Now,

\footnotetext{
'Here, the acronym 'sn' in the index stands for strongly normalizing.

${ }^{3}$ Note that $\sigma \downarrow$ is the normalized substitution defined by $(\sigma \downarrow)(x)=(\sigma x) \downarrow$.
} 
if $p_{i}$ is below the position $p$ of some variable $x$ in $l$ then we get $t_{i} \downarrow=(\Phi \circ \sigma)(l) / p_{i}$ since $t_{i}$ is also a maximal complete subterm of $\sigma x$. If $p_{i}$ is a non-variable position of $l$ then we have $t_{i}=\sigma\left(l / p_{i}\right)$. Since $t_{i}$ is complete, for every variable $x$ which occurs in $l$ (strictly) below $p_{i}$, $\sigma x$ is also complete. Let $\sigma^{\prime} x=(\Phi \circ \sigma)(x)=(\sigma x) \downarrow$ for these variables. By definition of $\Phi$ we get $t_{i} \downarrow=\sigma\left(l / p_{i}\right) \downarrow=\sigma^{\prime}\left(l / p_{i}\right) \downarrow$. We still have to show $\sigma^{\prime}\left(l / p_{i}\right) \downarrow=\sigma^{\prime}\left(l / p_{i}\right)$. From irreducibility of $\sigma^{\prime}$ and $\lambda<p_{i}$ we conclude that $\sigma^{\prime}\left(l / p_{i}\right)$ must be irreducible, because otherwise there would exist an inside critical pair in $\mathcal{R}$ by overlapping some rule into $l \rightarrow r$ properly below the root. Hence we are done.

Lemma 7 Suppose $\mathcal{R}$ is a locally confluent TRS. Let $l \rightarrow r$ be a rule of $\mathcal{R}$ such that there exists no inside critical pair by overlapping some other (or the same) rule of $\mathcal{R}$ into $l \rightarrow r$ properly below the root. Let $\sigma$ be a substitution such that $\sigma l$ is not complete. If $s=C[\sigma l] \rightarrow_{p, \sigma, l \rightarrow r} C[\sigma r]=t$ then $\Phi(s) \rightarrow^{+} \Phi(t)$.

Proof: Straightforward using Lemma 6.

Theorem 8 Let $\mathcal{R}_{b}, \mathcal{R}_{w}$ be terminating and confluent TRSs such that $\mathcal{R}_{b}$ is additionally $a$ non-collapsing overlay system. Then $\mathcal{R}=\mathcal{R}_{b} \oplus \mathcal{R}_{w}$ is terminating.

Proof: Suppose for a proof by contradiction that the disjoint union $\mathcal{R}=\mathcal{R}_{b} \oplus \mathcal{R}_{w}$ is non-terminating. Consider an infinite $(\mathcal{R}$-) derivation

$$
D: s_{1} \rightarrow s_{2} \rightarrow s_{3} \rightarrow \ldots
$$

with the additional minimality property that all proper subterms of $s_{1}$ are terminating and hence complete. Then $D$ must have the form

$$
D: s_{1} \rightarrow \ldots \rightarrow s_{n} \rightarrow_{\lambda} s_{n+1} \rightarrow \ldots,
$$

i.e., eventually some step $s_{n} \rightarrow_{\lambda} s_{n+1}$ is a (first) root reduction step. Clearly, this step is a $\rightarrow_{\neg \mathrm{sn}}$-step, and all proper subterms of $s_{n}$ are complete.

Now consider the case that $s_{n}$ is top white. All black principal subterms of $s_{n}$ are complete and any derivation issuing from them consists of (complete) top black reducts, since $\mathcal{R}_{b}$ is non-collapsing. Together with the infinity of $D$ this implies that all $s_{k}, k \geq n$, are top white and that $D$ contains infinitely many outer $\mathcal{R}_{w}$-steps. But then, by identifying abstraction of all black principal subterms (i.e., by their replacement by some same fresh variable), we obtain an infinite (pure) $\mathcal{R}_{w}$-derivation which contradicts termination of $\mathcal{R}_{w}$.

The other case is that $s_{n}$ is top black. Since all white principal subterms of $s_{n}$ are complete, all $\rightarrow \neg$ sn-steps in $D$ after $s_{n}$ (including $s_{n} \stackrel{\circ}{\rightarrow} s_{n+1}$ ) must be outer $\mathcal{R}_{b}$-steps, and there must be infinitely many of these. By definition of $\Phi$ we know that all principal subterms in $\Phi\left(s_{n}\right)$ are irreducible. Hence, applying $\Phi$ to $D$ and using Lemma 7 (which is applicable, because $\mathcal{R}_{b}$ is a locally confluent overlay system) and Lemma 5 we obtain the infinite $\mathcal{R}$-derivation

$$
\Phi\left(s_{n}\right) \rightarrow^{*} \Phi\left(s_{n+1}\right) \rightarrow^{*} \Phi\left(s_{n+2}\right) \rightarrow^{*} \ldots
$$


where all (proper) reduction steps are outer $\mathcal{R}_{b}$-steps. As above, identifying abstraction yields an infinite pure $\mathcal{R}_{b}$-derivation contradicting termination of $\mathcal{R}_{b}$. Thus we may conclude that $\mathcal{R}=\mathcal{R}_{b} \oplus \mathcal{R}_{w}$ must be terminating.

Let us give an example for illustrating the applicability of Theorem 8 .

Example 3 Consider the modified version of Example 1 where

$$
\mathcal{R}_{b}=\{f(a, b, x, x) \rightarrow f(x, x, x, x)\} \quad \mathcal{R}_{w}=\left\{\begin{aligned}
G(x, x) & \rightarrow x \\
G(A, B) & \rightarrow A \\
A & \rightarrow B
\end{aligned}\right\}
$$

Both systems are terminating and confluent, and moreover $\mathcal{R}_{b}$ is a non-collapsing overlay system. Hence, Theorem 8 yields completeness of the disjoint union $\mathcal{R}_{b} \oplus \mathcal{R}_{w}$. We note that none of the previous modularity results is applicable here (including the recent ones of [3]). In particular, $\mathcal{R}_{w}$ is neither overlaying nor left-linear, and $\mathcal{R}_{b}$ is also not left-linear.

\section{Asymmetric Versions of the Syntactic Approach}

Example 4 Consider the disjoint TRSs

$$
\mathcal{R}_{b}=\{f(x, g(x), y) \rightarrow f(y, y, y)\} \quad \mathcal{R}_{w}=\left\{\begin{array}{c}
H(x, x) \rightarrow K(B) \\
H(x, x) \rightarrow C \\
K(x) \rightarrow x
\end{array}\right\}
$$

Both systems are terminating as well as their disjoint union. However, we observe that none of the known modularity results applies here. In particular, both systems are not left-linear, and the second one is non-confluent (due to $B{ }^{+} \leftarrow H(x, x) \rightarrow C$ with $B$ and $C$ distinct normal forms).

Yet, we observe that collapsing reduction is deterministic in $\mathcal{R}_{w}$ above in the sense that whenever a term $s$ collapses to a variable $x$ then $x$ has a unique ancestor occurrence in $s$. This property is violated in Example 2 where we have for instance $K(z, z, z) \rightarrow z$, either by applying the rule $K(x, y, y) \rightarrow x$ (in which case the ancestor of $z$ in $K(z, z, z)$ is the first $z$ ) or by applying the rule $K(y, y, x) \rightarrow x$ (in which case the ancestor of $z$ in $K(z, z, z)$ is the last $z$ ). In fact, it turns out that also the property of not loosing the possibility of collapsing reductions is a crucial one. For instance, in the TRS $\{G(x) \rightarrow x, G(x) \rightarrow A\}$ we can collapse $G(x)$ to $x$ but after reduction of $G(x)$ to $A$ this possibility is lost.

In order to precisely define the first property informally described above we need some auxiliary definitions.

$\mathrm{RR} \mathbf{n}^{\circ} 3330$ 
Definition 9 For any labelled derivation $D: s \rightarrow^{*} t$ and any $q \in \mathcal{V} P$ os $(t)$ the set anc $(q, D)$ of ancestors of $q$ in $D$ is defined recursively as follows. Id $D$ is empty, then anc $(q, D)=\{q\}$. For a labelled one-step derivation $D: s \rightarrow_{p, \sigma, l \rightarrow r} t: \operatorname{anc}(q, D):=\{q\}$ if $p$ and $q$ are disjoint, and $\operatorname{anc}(q, D):=\left\{p p_{3} p_{2} \mid l / p_{3}=x\right\}$ if $q=p p_{1} p_{2}, r / p_{1}=x \in \mathcal{V}$ and $\sigma(x) / p_{2} \in \mathcal{V}$. For a non-empty labelled derivation $D=D_{1} ; D_{2}$ from $s$ to $t$ with $D_{1}: s \rightarrow^{*} s^{\prime}, D_{2}: s^{\prime} \rightarrow_{p, \sigma, l \rightarrow r} t$ we define: $\operatorname{anc}(q, D):=\bigcup_{q^{\prime} \in \operatorname{anc}\left(q, D_{2}\right)} \operatorname{anc}\left(q^{\prime}, D_{1}\right)$.

Slightly abusing notation, we denote $\bigcup_{D: s \rightarrow{ }^{*} t}$ anc $(q, D)$ (where the union ranges over all labelled derivations from $s$ to $t$ ) by anc $\left(q, s \rightarrow^{*} t\right)$. Furthermore, if $t=x$ (and consequently $q=\lambda)$, we also write - again slightly abusing notation - anc $(x, s)$ instead of anc $\left(\lambda, s \rightarrow^{*}\right.$ $x)$. If, for $t / q=x \in \mathcal{V}$, anc $\left(q, s \rightarrow^{*} t\right)=\{p\}$, then we say that $x$ in $t$ at (position) $q$ has a unique ancestor in $s$ at (position) $p$. If in this case $t=x$ (and consequently $q=\lambda$ ), we also write anc $(x, s)=p$ instead of anc $(x, s)=\{p\}$ and say that $x$ has a unique ancestor in $s$ at $p$.

In Example 2 e.g., for the derivation $D: K(x, x, x) \rightarrow_{K(x, y, y) \rightarrow x} x$ we have $\operatorname{anc}(\lambda, D)=$ $\{1\}$, and $\operatorname{anc}(x, K(x, x, x))=\{1,3\}$.

We remark that rewriting in left-linear TRSs enjoys some nice abstraction properties. In particular, we have the following.

Lemma 10 Let $\mathcal{R}$ be a left-linear TRS. If $s=C[x, \ldots, x]_{p_{1}, \ldots, p_{n}} \rightarrow^{*} x$ such that $p_{i} \in$ anc $(x, s)$, i.e., $p_{i} \in \operatorname{anc}(\lambda, D)$ for some labelled derivation $D: s \rightarrow^{*} x$, then $C\left[x_{1}, \ldots, x_{n}\right] \rightarrow^{*}$ $x_{i}$ (using the same rules at the same positions as in $D$ ) where $x_{1}, \ldots, x_{n}$ are mutually distinct fresh variables.

Proof: This is a consequence of the left-linearity of $\mathcal{R}$.

Definition $11 A T R S \mathcal{R}^{\mathcal{F}}$ is said to be uniquely collapsing (UC) if, for every $s \in \mathcal{T}(\mathcal{F},\{x\})$, $s=C[x, \ldots, x]_{p_{1}, \ldots, p_{n}} \rightarrow^{*} x$ (where all occurrences of $x$ in $s$ are displayed) implies that $x$ has a unique ancestor in $s$ at $p_{i}$ (for some unique $i, 1 \leq i \leq n$ ). We say that $\mathcal{R}^{\mathcal{F}}$ is collapsing confluent (CCR) if $x^{*} \leftarrow s \rightarrow^{*} t$ implies $t \rightarrow^{*} x$ for every $s, t \in \mathcal{T}(\mathcal{F}, \mathcal{V}), x \in \mathcal{V}$.

We observe that collapsing confluence is a restricted version of the normal form property NF (where the only normal forms considered are variables), and can also be expressed more locally as follows.

Lemma 12 For any $T R S \mathcal{R}^{\mathcal{F}}$ the following assertions are equivalent:

(1) $\forall s, t \in \mathcal{T}(\mathcal{F}, \mathcal{V}), x \in \mathcal{V}: x^{*} \leftarrow s \rightarrow t \Longrightarrow t \rightarrow^{*} x$.

(2) $\forall s, t \in \mathcal{T}(\mathcal{F}, \mathcal{V}), x \in \mathcal{V}: x^{*} \leftarrow s \rightarrow^{*} t \Longrightarrow t \rightarrow^{*} x$.

(3) $\forall t \in \mathcal{T}(\mathcal{F}, \mathcal{V}), x \in \mathcal{V}: x \leftrightarrow^{*} t \Longrightarrow t \rightarrow^{*} x$.

INRIA 
Proof: Straightforward. For the implication (1) $\Longrightarrow(2)$, assuming (1), one shows

$$
\forall n \forall s, t \in \mathcal{T}(\mathcal{F}, \mathcal{V}), x \in \mathcal{V}: x^{*} \leftarrow s \rightarrow^{n} t \Longrightarrow t \rightarrow^{*} x
$$

by a straightforward induction on $n$. Analogously, for the implication (1) $\Longrightarrow(3)$, assuming (1), one shows

$$
\forall n \forall s, t \in \mathcal{T}(\mathcal{F}, \mathcal{V}), x \in \mathcal{V}: x \leftrightarrow^{n} t \Longrightarrow t \rightarrow^{*} x
$$

by a straightforward induction on $n$. The implications $(2) \Longrightarrow(1)$ and $(3) \Longrightarrow(1)$ obviously hold. Hence, all three properties are equivalent.

Next we define a transformation which enables to abstract from white 'layers' but keeps the information concerning potential (white) 'layer collapses'.

Definition 13 Let $\mathcal{R}_{b}, \mathcal{R}_{w}$ be disjoint TRSs such that $\mathcal{R}_{w}$ is uniquely collapsing. The (white) abstraction mapping $\Theta: \mathcal{T}\left(\mathcal{F}_{b} \uplus \mathcal{F}_{w}\right) \rightarrow \mathcal{T}\left(\mathcal{F}_{b} \uplus\left\{G^{1}, A^{0}\right\}\right)$ is recursively defined as follows:

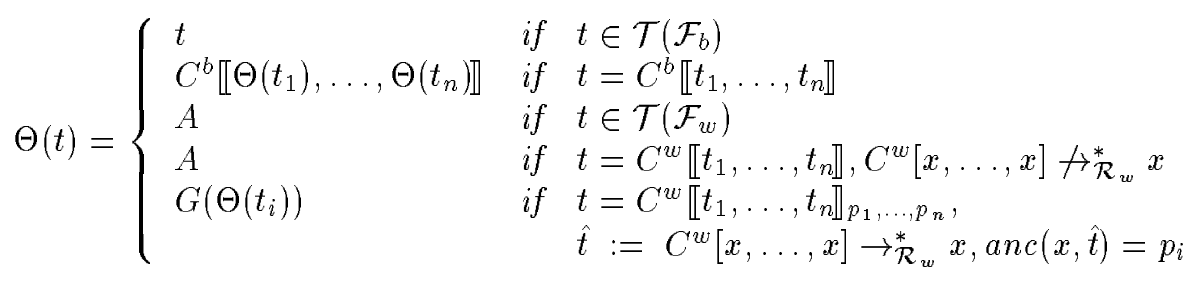

We observe that $\Theta$ above is well-defined, since $\mathcal{R}_{w}$ is assumed to be uniquely collapsing. For illustration, consider in Example 4 the derivation

$f(K(H(a, a)), g(H(a, a)), K(b)) \rightarrow_{\mathcal{R}_{w}} f(H(a, a), g(H(a, a)), K(b)) \rightarrow_{\mathcal{R}_{b}} f(K(b), K(b), K(b))$

(where $a, b$ are assumed to be black constants). Here, abstraction with $\Theta$ yields:

$$
f(A, g(A), G(b))=f(A, g(A), G(b)) \rightarrow_{\mathcal{R}_{b}} f(G(b), G(b), G(b)) .
$$

Lemma 14 Let $\mathcal{R}_{b}, \mathcal{R}_{w}$ be disjoint TRSs such that $\mathcal{R}_{b}$ is non-collapsing and $\mathcal{R}_{w}$ is uniquely collapsing and collapsing confluent. Let s be a top black term. Then the following properties hold:

(a) $s \stackrel{o}{\rightarrow}_{\mathcal{R}_{b}} t \Longrightarrow \Theta(s) \rightarrow_{\mathcal{R}_{b}} \Theta(t)$.

(b) $s \stackrel{i}{\rightarrow}_{\mathcal{R}_{b}} t \Longrightarrow \Theta(s) \rightarrow \overline{\overline{\mathcal{R}}}_{b} \Theta(t)$.

(c) $s \stackrel{i}{\rightarrow}_{\mathcal{R}_{w}} t \Longrightarrow \Theta(s) \rightarrow \overline{\overline{\{G}}_{(x) \rightarrow x\}} \Theta(t)$.

$\operatorname{RR} \mathbf{n}^{\circ} 3330$ 
Proof: (a) is straightforward by definition of $\Theta$. (b) is proved by induction on $n=\operatorname{rank}(s)$ and case analysis using the non-collapsing property of $\mathcal{R}_{b}$ and (a). The proof of (c) is also by induction on $n=\operatorname{rank}(s)$ and case analysis exploiting that $\mathcal{R}_{w}$ is uniquely collapsing and collapsing confluent. Note that without collapsing confluence of $\mathcal{R}_{w}$ an inner $\mathcal{R}_{w}$-step could be translated into a step using the rule $G(x) \rightarrow A$. This is due to the fact that in a top white, white term $C^{w}[x, \ldots, x]$ the possibility of a collapse to $x$ might be eliminated by a next (possibly non-collapsing) $\mathcal{R}_{w}$-step.

Lemma $15 A$ TRS $\mathcal{R}$ is terminating if and only if $\mathcal{R} \oplus\{G(x) \rightarrow x\}$ is terminating.

Proof: The 'if-part' of the equivalence is trivial. The 'only-if' part is a consequence of [16, Theorem 6].

An alternate (direct and easy) proof (of the 'only-if' part) is obtained by considering the interpretation $\rho$ of terms of $\mathcal{T}(\mathcal{F} \uplus\{G\}, \mathcal{V})$ in the term algebra $\mathcal{T}(\mathcal{F}, \mathcal{V})$ which interprets every $f \in \mathcal{F}$ by itself, and $G$ as identity function. Since any infinite $(\mathcal{R} \oplus\{G(x) \rightarrow x\})$ derivation must contain infinitely many $\mathcal{R}$-steps, non-termination of $\mathcal{R} \oplus\{G(x) \rightarrow x\}$ would imply non-termination of $\mathcal{R}$, simply by applying $\rho$.

A slightly more sophisticated interpretation which yields a proper decrease for any (proper) reduction step in $\mathcal{R} \oplus\{G(x) \rightarrow x\}$ is as follows (assuming termination of $\mathcal{R}$ ): Take as semantic domain for $\mathcal{F} \uplus\{G\}: A=\mathcal{T}(\mathcal{F}, \mathcal{V}) \times N$ at, with $N$ at being the set of natural numbers. This yields a well-founded monotone $(\mathcal{F} \uplus\{G\})$-algebra $(A,>)$ by defining $f_{A}\left(\left(t_{1}, n_{1}\right), \ldots,\left(t_{k}, n_{k}\right)\right)=\left(f\left(t_{1}, \ldots, t_{k}\right), n_{1}+\ldots+n_{k}\right)$ for any $f \in \mathcal{F}$, and $G_{A}((t, n))=$ $(t, n+1)$, and taking $>$ to be the well-founded lexicographic combination of $\rightarrow_{\mathcal{R}}^{+}$and $>_{N a t}$ (the usual ordering on natural numbers). Clearly, $(A,>)$ is compatible with $\mathcal{R} \oplus\{G(x) \rightarrow x\}$, cf. e.g. [26], hence $\mathcal{R} \oplus\{G(x) \rightarrow x\}$ is terminating.

Theorem 16 Let $\mathcal{R}_{b}, \mathcal{R}_{w}$ be two disjoint terminating TRSs. If $\mathcal{R}_{b}$ is non-collapsing and $\mathcal{R}_{w}$ is uniquely collapsing and collapsing confluent, then $\mathcal{R}_{b} \oplus \mathcal{R}_{w}$ is terminating.

Proof: For a proof by contradiction assume $\mathcal{R}_{b}$ and $\mathcal{R}_{w}$ are given as above such that $\mathcal{R}^{\mathcal{F}}=\mathcal{R}_{b} \oplus \mathcal{R}_{w}$ is non-terminating. We consider a counterexample of minimal rank

$$
D: s_{0} \rightarrow_{\mathcal{R}} s_{1} \rightarrow_{\mathcal{R}} s_{2} \rightarrow_{\mathcal{R}} \ldots
$$

where w.l.o.g. we may assume $s_{i} \in \mathcal{T}(\mathcal{F})=\mathcal{T}\left(\mathcal{F}_{b} \uplus \mathcal{F}_{w}\right)$ for all $i, 0 \leq i$. Since $D$ is minimal and $\mathcal{R}_{b}$ is non-collapsing we conclude that all $s_{i}$ are top black (otherwise, i.e., if all $s_{i}$ were top white, identifying abstraction of the principal subterms (i.e., their replacement by some fresh variable) in $D$ would yield an infinite $\mathcal{R}_{w}$-derivation contradicting termination of $\mathcal{R}_{w}$ ). Now, applying Lemma 14 and exploiting the fact that $D$ contains infinitely many outer $\mathcal{R}_{b}$-steps we obtain an infinite $\left(\mathcal{R}_{b} \oplus\{G(x) \rightarrow x\}\right)$-derivation

$$
\Theta(D): \Theta\left(s_{0}\right) \rightarrow^{*} \Theta\left(s_{1}\right) \rightarrow^{*} \Theta\left(s_{2}\right) \rightarrow^{*} \ldots
$$

But by Lemma 15 this implies non-termination of $\mathcal{R}_{b}$, hence a contradiction. 
In Example 4 the system $\mathcal{R}_{b}=\{f(x, g(x), y) \rightarrow f(y, y, y)\}$ is non-collapsing and $\mathcal{R}_{w}=$ $\{H(x, x) \rightarrow K(B), H(x, x) \rightarrow C, K(x) \rightarrow x\}$ is easily shown to be both uniquely collapsing and collapsing confluent. Hence, by Theorem 16 we conclude termination of $\mathcal{R}_{b} \oplus \mathcal{R}_{w}$.

Example 5 Consider the disjoint TRSs

$$
\mathcal{R}_{b}=\{f(a, g(x), y) \rightarrow f(y, y, y)\} \quad \mathcal{R}_{w}=\left\{\begin{array}{c}
H(x, x) \rightarrow K(B) \\
K(x) \rightarrow C \\
K(x) \rightarrow x
\end{array}\right\}
$$

Both systems are terminating as well as their disjoint union. However, we observe that none of the known modularity results (including Theorem 16 above) applies here. In particular, the second system is not left-linear and not (collapsing) confluent (due to $x \leftarrow K(x) \rightarrow C$ with $C$ irreducible). Observe, however, that in contrast to Example 4 the non-collapsing system $\mathcal{R}_{b}$ is additionally left-linear.

We shall show now that dropping the collapsing confluence condition for $\mathcal{R}_{w}$ in Theorem 16 is possible provided we additionally require that $\mathcal{R}_{b}$ is left-linear.

Lemma 17 Let $\mathcal{R}_{b}, \mathcal{R}_{w}$ be disjoint TRSs such that $\mathcal{R}_{b}$ is non-collapsing and $\mathcal{R}_{w}$ is uniquely collapsing. Let $s$ be a top black term. Then the following properties hold:

(a) $s \stackrel{o}{\rightarrow}_{\mathcal{R}_{b}} t \Longrightarrow \Theta(s) \rightarrow_{\mathcal{R}_{b}} \Theta(t)$.

(b) $s \stackrel{i}{\rightarrow}_{\mathcal{R}_{b}} t \Longrightarrow \Theta(s) \rightarrow \overline{\overline{\mathcal{R}}}_{b} \Theta(t)$.

(c) $s \stackrel{i}{\rightarrow}_{\mathcal{R}_{w}} t \Longrightarrow \Theta(s) \rightarrow{ }_{\{G(x) \rightarrow x, G(x) \rightarrow A\}} \Theta(t)$.

Proof: (a) and (b) hold by Lemma 14(a) and (b). (c) follows by induction on rank(s) (cf. the proof of Lemma 14(c)). In particular, collapsing confluence of $\mathcal{R}_{w}$ is not needed here.

Lemma 18 Let $\mathcal{R}$ be a left-linear TRS. Then, $\mathcal{R}$ is terminating if and only if $\mathcal{R} \oplus\{G(x) \rightarrow$ $x, G(x) \rightarrow A\}$ is terminating.

Proof: The 'if-part' of the equivalence is trivial. For the 'only-if' part assume that $\mathcal{R}$ is terminating. Let $\mathcal{R}_{G_{x}}:=\{G(x) \rightarrow x\}, \mathcal{R}_{G_{A}}:=\{G(x) \rightarrow A\}$. Obviously, $\mathcal{R}_{G_{A}}$ is terminating. By assumption and Lemma 15 we know that $\left(\mathcal{R} \cup \mathcal{R}_{G_{x}}\right)$ is terminating. Hence, using the quasi-commutation approach of Bachmair \& Dershowitz [1, Lemmas 1,2], for termination of $\mathcal{R} \cup\left(\mathcal{R}_{G_{x}} \cup \mathcal{R}_{G_{A}}\right)=\left(\mathcal{R} \cup \mathcal{R}_{G_{x}}\right) \cup \mathcal{R}_{G_{A}}$ it suffices to show that $\left(\mathcal{R} \cup \mathcal{R}_{G_{x}}\right)$

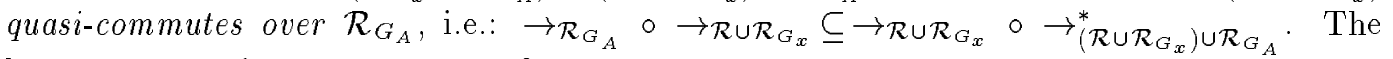
latter property is a consequence of

(1) $\rightarrow_{\mathcal{R}_{G_{A}}} \circ \rightarrow \mathcal{R}_{G_{x}} \subseteq \rightarrow_{\mathcal{R}_{G_{x}}} \circ \rightarrow_{\mathcal{R}_{G_{A}}}$, and

$\operatorname{RR} \mathbf{n}^{\circ} 3330$ 


$$
(2) \rightarrow_{\mathcal{R}_{G_{A}}} \circ \rightarrow_{\mathcal{R}} \subseteq \rightarrow_{\mathcal{R}} \circ \rightarrow_{\mathcal{R}_{G_{A}}}^{*} \cdot 4
$$

Now, (1) is straightforward by an easy case analysis exploiting the special shape of the rules $G(x) \rightarrow x$ and $G(x) \rightarrow A$. (2) is also easy by a standard case analysis, but essentially relies on left-linearity of $\mathcal{R}$. Hence, we are done.

Theorem 19 Let $\mathcal{R}_{b}, \mathcal{R}_{w}$ be two disjoint terminating TRSs. If $\mathcal{R}_{b}$ is non-collapsing and left-linear, and $\mathcal{R}_{w}$ is uniquely collapsing, then $\mathcal{R}_{b} \oplus \mathcal{R}_{w}$ is terminating.

Proof: For a proof by contradiction assume $\mathcal{R}_{b}$ and $\mathcal{R}_{w}$ are given as above such that $\mathcal{R}=\mathcal{R}_{b} \oplus \mathcal{R}_{w}$ is non-terminating. We consider a counterexample of minimal rank

$$
D: s_{0} \rightarrow_{\mathcal{R}} s_{1} \rightarrow_{\mathcal{R}} s_{2} \rightarrow_{\mathcal{R}} \ldots
$$

where w.l.o.g. we may assume $s_{i} \in \mathcal{T}(\mathcal{F})=\mathcal{T}\left(\mathcal{F}_{b} \uplus \mathcal{F}_{w}\right)$ for all $i, 0 \leq i$. Since $D$ is minimal and $\mathcal{R}_{b}$ is non-collapsing we conclude that all $s_{i}$ are top black (otherwise, i.e., if all $s_{i}$ were top white, identifying abstraction of the principal subterms in $D$ would yield an infinite $\mathcal{R}_{w}$-derivation contradicting termination of $\mathcal{R}_{w}$ ). Now, applying Lemma 17 and exploiting the fact that $D$ contains infinitely many outer $\mathcal{R}_{b}$-steps we obtain an infinite $\left(\mathcal{R}_{b} \oplus\{G(x) \rightarrow x, G(x) \rightarrow A\}\right)$-derivation

$$
\Theta(D): \Theta\left(s_{0}\right) \rightarrow^{*} \Theta\left(s_{1}\right) \rightarrow^{*} \Theta\left(s_{2}\right) \rightarrow^{*} \ldots
$$

But by left-linearity of $\mathcal{R}_{b}$ and Lemma 18 this implies non-termination of $\mathcal{R}_{b}$, hence a contradiction.

Using Theorem 19 it is straightforward to prove termination of $\left(\mathcal{R}_{b} \oplus \mathcal{R}_{w}\right)$ in Example 5: $\mathcal{R}_{b}=\{f(a, g(x), y) \rightarrow f(y, y, y)\}$ is non-collapsing and left-linear, and $\mathcal{R}_{w}=\{H(x, x) \rightarrow$ $K(B), K(x) \rightarrow C, K(x) \rightarrow x\}$ is easily shown to be uniquely collapsing. Hence Theorem 19 is applicable.

However, let us remark that in general applying the preservation criteria for termination that rely on the properties UC and CCR, i.e., Theorems 16 and 19, is not obvious. In fact, the properties UC and CCR are undecidable in general, even for left-linear terminating TRSs (this can be shown by using the undecidability of PCP, Post's correspondence problem). Consequently, it might be worthwhile looking for interesting decidable (syntactic) conditions ensuring them (this seems to be non-trivial). Yet, we think that the analysis performed is of independent interest because it provides a deeper structural insight into the crucial phenomena causing (non-)termination in disjoint unions of terminating systems.

\footnotetext{
${ }^{4}$ In fact, in the latter reduction here instead of $\rightarrow_{G_{A}}^{*}$ one parallel reduction step using (instances of) the $\mathcal{R}_{G_{x}}$-rule $G(x) \rightarrow A$ suffices.
} 


\section{Modularity Criteria for UC and CCR}

Next we shall investigate how the newly introduced properties of being uniquely collapsing (UC) and collapsing confluent (CCR) relate to other well-known confluence, normal form and consistency properties. Moreover, we shall study their modularity behaviour.

Lemma 20 For any TRS the following implications hold and are proper:

(a) $N F \Longrightarrow C C R$.

(b) $\mathrm{CR} \Longrightarrow \mathrm{CCR} \Longrightarrow \mathrm{CON}$.

(c) $U C \Longrightarrow C O N \rightarrow$

Proof: The implication in (a) follows from the respective definitions (and Lemma 12). Furthermore, the TRS $\{a \rightarrow b, a \rightarrow c\}$ (over the signature $\mathcal{F}=\{a, b, c\}$ ) is a counterexample to the reverse implication. In (b), the implications also follow from the respective definitions (and making use of Lemma 12). The implications are proper, because for instance $\{g(x) \rightarrow x, g(a) \rightarrow b\}$ is CCR but not CR, and $\{f(x) \rightarrow x, f(x) \rightarrow g(x)\}$ is CON but not CCR. Finally, the implication UC $\Longrightarrow \mathrm{CON}^{\rightarrow}$ in (c) holds, since any counterexample $x^{+} \leftarrow C[x, y]_{p_{1}, p_{2}} \rightarrow^{+} y(x \neq y)$ to $\mathrm{CON}^{\rightarrow}$ immediately yields a counterexample to UC: $x+\leftarrow C[x, x]_{p_{1}, p_{2}} \rightarrow^{+} x$ where $p_{1}, p_{2}$ are distinct ancestors of $x$ in $C[x, x]$. Moreover, $\{f(x, x) \rightarrow x\}$ is obviously $\mathrm{CON}^{\rightarrow}$ but not $\mathrm{UC}$.

Neither UC nor CCR is a modular property of TRSs as shown by the following examples.

Example 6 The disjoint TRSs

$$
\mathcal{R}_{b}=\left\{\begin{array}{r}
f(x, x, y, z) \rightarrow y \\
f(x, g(x), y, z) \rightarrow z
\end{array}\right\} \quad \mathcal{R}_{w}=\left\{\begin{array}{l}
G(x) \rightarrow x \\
G(x) \rightarrow A
\end{array}\right\}
$$

are both uniquely collapsing (as is not difficult to verify). However, their disjoint union $\mathcal{R}=\mathcal{R}_{b} \oplus \mathcal{R}_{w}$ is not even consistent w.r.t. reduction since in $\mathcal{R}$ we have for instance:

$$
z \leftarrow f(A, g(A), y, z){ }^{+} \leftarrow f(G(g(A)), G(g(A)), y, z) \rightarrow y .
$$

Example 7 The disjoint TRSs

$$
\mathcal{R}_{b}=\{f(x, x, y) \rightarrow y\} \quad \mathcal{R}_{w}=\left\{\begin{array}{l}
A \rightarrow B \\
A \rightarrow C
\end{array}\right\}
$$

are obviously collapsing confluent, but in $\mathcal{R}=\left(\mathcal{R}_{b} \oplus \mathcal{R}_{w}\right)$ we have

$$
f(B, C, y){ }^{+} \leftarrow f(A, A, y) \rightarrow y
$$

where $f(B, C, y)$ and $y$ are distinct normal forms. Hence, $\mathcal{R}$ is not collapsing confluent.

$\mathrm{RR} \mathbf{n}^{\circ} 3330$ 


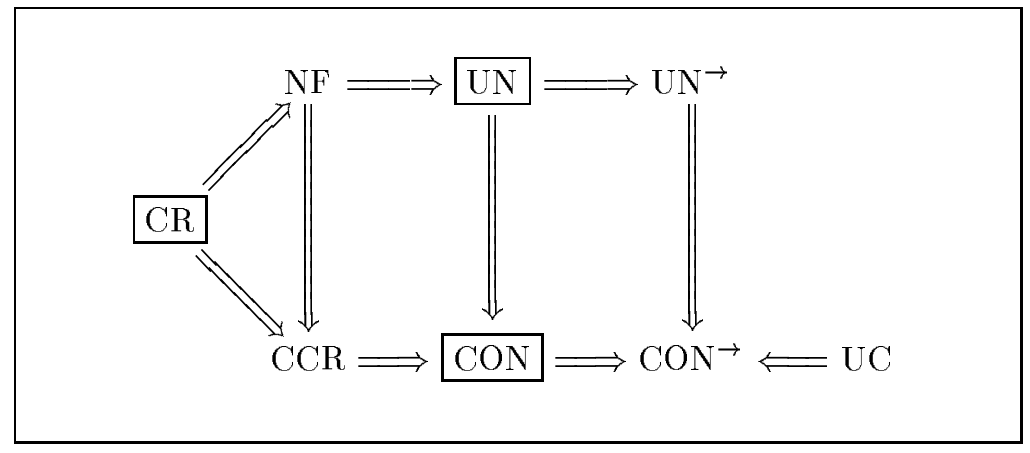

Figure 1: confluence / normal form properties and their modularity behaviour

Figure 1 summarizes the relationships between the various confluence and normal form properties considered as well as their modularity behaviour. Missing implications do not hold, the boxed properties are modular and the others are not modular (cf. [24], [21], [15], [13], [22, 14] for the relations and (non-)modularity properties not treated above).

The properties NF and $\mathrm{UN}^{\rightarrow}$ are not modular as shown by Middeldorp ([15]). However, modularity can be recovered by imposing left-linearity (LL): NF $\wedge$ LL is modular ([15]), and $\mathrm{UN}^{\rightarrow} \wedge \mathrm{LL}$ as well as $\mathrm{CON}^{\rightarrow} \wedge \mathrm{LL}$ are modular ([13]). Interestingly, it turns out that for collapsing confluence (CCR) and the property of being uniquely collapsing (UC) modularity can also be recovered by imposing left-linearity.

Theorem 21 Collapsing confluence is modular for left-linear TRSs (i.e., CCR $\wedge L$ is modular).

Proof: For the non-trivial part of the modularity statement assume that $\mathcal{R}_{b}$ and $\mathcal{R}_{w}$ are disjoint left-linear TRSs that are collapsing confluent. First we observe that, by [21, Lemma $3.6] \leftrightarrow_{\mathcal{R}^{\mathcal{T}}}^{*}$ is a conservative extension of both $\leftrightarrow_{\mathcal{R}_{b}}^{*}$ and $\leftrightarrow_{\mathcal{R}^{w}}^{*}$, i.e., for any $i \in\{b, w\}$ :

$$
(*) \quad \forall s, t \in \mathcal{T}\left(\mathcal{F}_{i}, \mathcal{V}\right): s \leftrightarrow_{\mathcal{R}_{i}}^{*} t \Longleftrightarrow s \leftrightarrow_{\mathcal{R}}^{*} t
$$

Now assume that in $\mathcal{R}=\mathcal{R}_{b} \oplus \mathcal{R}_{w}$ we have $x \underset{\mathcal{R}}{*} \leftarrow s \rightarrow_{\mathcal{R}}^{*} t$. Then we must show: $t \rightarrow_{\mathcal{R}}^{*} x$. To this end we first reduce $t$ to an inner preserved reduct as follows: If $t \in \mathcal{T}\left(\mathcal{F}_{i}, \mathcal{V}\right)$ (for some $i \in$ $\{b, w\})$ then $t$ is already inner preserved. In that case we get $x \leftrightarrow_{\mathcal{R}_{i}}^{*} t$ by $(*)$, and collapsing confluence of $\mathcal{R}_{i}$ implies $t \rightarrow_{\mathcal{R}_{i}}^{*} x$ as desired. Otherwise, assume w.l.o.g. $t=C^{b} \llbracket t_{1}, \ldots, t_{n} \rrbracket$. Since every term has a preserved reduct (cf. [11]), every principal subterm $t_{i}$ of $t$ can be reduced to a preserved reduct $t_{i}^{\prime}$. Hence we get $t=C^{b} \llbracket t_{1}, \ldots, t_{n} \rrbracket \rightarrow_{\mathcal{R}}^{*} C^{b}\left[t_{1}^{\prime}, \ldots, t_{n}^{\prime}\right]=: t^{\prime}$ and $x \leftrightarrow^{*} t^{\prime}$. Obviously, $t^{\prime}$ is of the form $t^{\prime}=D^{b} \llbracket u_{1}, \ldots, u_{m} \rrbracket$ and is inner preserved. Now, according to [21, Lemma 3.13], an abstraction (modulo $\leftrightarrow_{\mathcal{R}}^{*}$ ) of the maximal top white special subterms in $x$ and $t^{\prime}$ yields the following:

$$
x \leftrightarrow_{\mathcal{R}_{b}}^{*} D^{b}\left[x_{1}, \ldots, x_{m}\right]
$$


where $x_{1}, \ldots, x_{m}$ denote (not necessarily distinct) fresh variables with $x_{j}=x_{k} \Longleftrightarrow u_{j} \leftrightarrow_{\mathcal{R}}^{*}$ $u_{k}$, for $1 \leq j, k \leq m .{ }^{5}$ Thus, by collapsing confluence of $\mathcal{R}_{b}$ we get

$$
D^{b}\left[x_{1}, \ldots, x_{m}\right] \rightarrow_{\mathcal{R}_{b}}^{*} x .
$$

Since $\mathcal{R}_{b}$ is left-linear (and $x$ does not occur among the $x_{i}$ ), by Lemma 10 we can replace the variable occurrences $x_{1}, \ldots, x_{m}$ by mutually distinct fresh variables $y_{1}, \ldots, y_{m}$ such that

$$
D^{b}\left[y_{1}, \ldots, y_{m}\right] \rightarrow_{\mathcal{R}_{b}}^{*} x
$$

(using the same rules at the same positions). ${ }^{6}$ From the latter derivation we finally obtain by instantiation

$$
D^{b}\left[u_{1}, \ldots, u_{m}\right] \rightarrow_{\mathcal{R}_{b}}^{*} x
$$

which together with $t \rightarrow_{\mathcal{R}}^{*} D^{b}\left[u_{1}, \ldots, u_{m}\right]$ yields

$$
t \rightarrow_{\mathcal{R}}^{*} x
$$

as desired. Hence we are done.

In order to illustrate what may go wrong in the above construction for non-left-linear systems consider again Example 7. There we had the derivations

$$
f(B, C, y)_{\mathcal{R}}^{*} \leftarrow f(A, A, y) \rightarrow_{\mathcal{R}} y
$$

in the disjoint union. Now, $f(B, C, y)$ is inner preserved (it is even irreducible). Hence, abstraction (modulo $\leftrightarrow_{\mathcal{R}}^{*}$ ) yields (due to $B \leftrightarrow_{\mathcal{R}}^{*} C$ )

$$
f(z, z, y) \leftrightarrow_{\mathcal{R}_{b}}^{*} y
$$

with $z$ a fresh variable. Collapsing confluence of $\mathcal{R}_{b}=\{f(x, x, y) \rightarrow y\}$ entails $f(z, z, y) \rightarrow_{\mathcal{R}_{b}}^{*} y$, in fact even

$$
f(z, z, y) \rightarrow_{\mathcal{R}_{b}} y .
$$

But now linearization w.r.t. $z$ is impossible, i.e., $f\left(z_{1}, z_{2}, y\right) \rightarrow_{\mathcal{R}_{b}} y$ (from which we would get $f(B, C, y) \rightarrow_{\mathcal{R}_{b}} y$ as desired) does not hold.

Theorem 22 A left-linear TRSs is uniquely collapsing if and only it is consistent w.r.t. reduction ( $L L \Longrightarrow[U C \Longleftrightarrow C O N \rightarrow]$ ).

\footnotetext{
${ }^{5}$ Note that in order to ensure applicability of [21, Lemma 3.13] as above one has to verify that - in the terminology of [21] $-t^{\prime}$ is ' $\leftrightarrow \mathcal{R}_{b}^{*}$-normalized' and $u_{1}, \ldots, u_{m}$ are ' $\leftrightarrow \mathcal{R}^{*}$-normalized'. The latter property (which implies the former one) follows from the preservation of the $u_{j}$ in combination with collapsing confluence of $\mathcal{R}_{b}$ and of $\mathcal{R}_{w}$.

${ }^{6}$ Note that this 'linearization' of the derivation essentially relies on left-linearity of $\mathcal{R}_{b}$ !
}

$\operatorname{RR} \mathbf{n}^{\circ} 3330$ 
Proof: Assume $\mathcal{R}$ is left-linear. The implication $\mathrm{UC} \Longrightarrow \mathrm{CON}^{\rightarrow}$ holds (even without leftlinearity) by Lemma 20 (c). Conversely, assuming $\neg$ UC we have to show $\neg \mathrm{CON}^{\rightarrow}$. Hence, consider a counterexample (to UC), i.e., a derivation

$$
s=C[x \ldots, x]_{p_{1}, \ldots, p_{n}} \rightarrow^{*} x
$$

(with $n \geq 2$ ) where $x$ has two distinct ancestor occurrences $p_{i}$ and $p_{j}$ in $s$, let's say $p_{1}$ and $p_{n}$. By left-linearity of $\mathcal{R}$ and Lemma 10 this implies

$$
C[y, x, \ldots, x, z] \rightarrow^{*} y
$$

and

$$
C[y, x, \ldots, x, z] \rightarrow^{*} z
$$

(for some fresh distinct variables $y, z$ ) which yields $\neg \mathrm{CON}^{\rightarrow}$ as desired.

In view of Theorem 22, and since confluence implies in particular consistency w.r.t. reduction and collapsing confluence, Theorem 16 above constitutes a generalization of Theorem 4 (due to Toyama, Klop \& Barendregt [25]). Furthermore we remark that together with the (nontrivial) modularity of $\mathrm{CON}^{\rightarrow} \wedge$ LL which was proved in [13] Theorem 22 above entails the following consequence.

Theorem 23 Being uniquely collapsing is modular for left-linear TRSs (i.e., UCALL is modular).

\section{Extensions to Non-Disjoint Unions}

Extensions of our asymmetric preservation results for termination are possible for instance for composable TRS ([17, 18, 20]) where constructors may be shared as well as the defining rules for all shared defined symbols. In such combinations the problematic 'layer-coalescing' reductions are not only possible by application of collapsing rules, but also by application of 'shared function symbol lifting' rules. Taking this effect into account, Theorem 8 extends in a natural way to composable TRSs by requiring 'layer-preservation' ([20]) instead of the non-collapsing property of one of the involved systems (cf. [9, Theorem 5.4.12]). Similar extensions (to composable systems) of the presented symmetric and asymmetric preservation results for the syntactic approach seem also possible along the same line of reasoning by replacing the non-collapsing requirement by layer-preservation and by forbidding shared function symbol lifting rules. Whether extensions to certain hierarchical combinations are possible remains to be clarified (cf. e.g. [12], [2], [6]). 


\section{References}

[1] L. Bachmair and N. Dershowitz. Commutation, transformation, and termination. In J. Siekmann, ed., Proc. 8th Int. Conf. on Automated Deduction (CADE'86), LNCS 230, pp. 5-20. Springer, 1986.

[2] N. Dershowitz. Hierarchical termination. In N. Dershowitz and N. Lindenstrauss, eds., Proc. 4th CTRS (1994), LNCS 968, pp. 89-105. Springer, 1995.

[3] N. Dershowitz. Innocuous constructor-sharing combinations. In H. Comon, ed., Proc. 8th Int. Conf. on Rewriting Techniques and Applications (RTA'97), LNCS 1232, pp. 202-216. Springer, 1997.

[4] N. Dershowitz and J.-P. Jouannaud. Rewrite systems. In J. van Leeuwen, ed., Formal models and semantics, Handbook of Theoretical Computer Science, volume B, chapter 6, pp. 243-320. Elsevier - The MIT Press, 1990.

[5] K. Drosten. Termersetzungssysteme. Informatik-Fachberichte 210. Springer, 1989. In German.

[6] M. Fernández and J.-P. Jouannaud. Modular termination of term rewriting systems revisited. In E. Astesiano, G. Reggio, and A. Tarlecki, eds., Recent Trends in Data Type Specification, LNCS 906, pp. 255-272, Springer, 1995. (10th Workshop on Specification of Abstract Data Types Joint with the 5th COMPASS Workshop, selected papers).

[7] B. Gramlich. Generalized sufficient conditions for modular termination of rewriting. Applicable Algebra in Engineering, Communication and Computing, 5:131-158, 1994.

[8] B. Gramlich. Abstract relations between restricted termination and confluence properties of rewrite systems. Fundamenta Informaticae, 24:3-23, 1995.

[9] B. Gramlich. Termination and Confluence Properties of Structured Rewrite Systems. $\mathrm{PhD}$ thesis, Fachbereich Informatik, Universität Kaiserslautern, Jan. 1996.

[10] J. W. Klop. Term rewriting systems. In S. Abramsky, D. Gabbay, and T. Maibaum, eds., Handbook of Logic in Computer Science, volume 2, chapter 1, pp. 2-117. Clarendon Press, Oxford, 1992.

[11] J. W. Klop, A. Middeldorp, Y. Toyama, and R. Vrijer. Modularity of confluence: A simplified proof. Information Processing Letters, 49:101-109, 1994.

[12] M.R.K. Krishna Rao. Modular proofs for completeness of hierarchical term rewriting systems. Theoretical Computer Science, 151(2):487-512, Nov. 1995.

[13] M. Marchiori. Modularity of $\mathbf{U N}^{\rightarrow}$ for left-linear term rewriting systems. Technical Report CS-R9433, CWI, Amsterdam, May 1994.

$\operatorname{RR} \mathbf{n}^{\circ} 3330$ 
[14] M. Marchiori. Modularity of Completeness Revisited . In J. Hsiang, ed., Proc. 6th Int. Conf. on Rewriting Techniques and Applications (RTA'95), LNCS 914, pp. 2-10. Springer, 1995.

[15] A. Middeldorp. Modular Properties of Term Rewriting Systems. PhD thesis, Free University, Amsterdam, 1990.

[16] A. Middeldorp, H.Ohsaki and H. Zantema. Transforming termination by self-labelling. In M.A. McRobbie and J.K. Slaney, eds., Proc. 13th Int. Conf. on Automated Deduction (CADE'96), LNAI 1104, pp. 373-387. Springer, 1996.

[17] A. Middeldorp and Y. Toyama. Completeness of combinations of constructor systems. Journal of Symbolic Computation, 15:331-348, Sept. 1993.

[18] E. Ohlebusch. Modular Properties of Composable Term Rewriting Systems. PhD thesis, Universität Bielefeld, 1994. Report 94-01.

[19] E. Ohlebusch. On the modularity of termination of term rewriting systems. Theoretical Computer Science, 136:333-360, 1994.

[20] E. Ohlebusch. Modular properties of composable term rewriting systems. Journal of Symbolic Computation, 20(1):1-42, 1995.

[21] M. Schmidt-Schauß. Unification in a combination of arbitrary disjoint equational theories. Journal of Symbolic Computation, 8(1):51-99, 1989.

[22] M. Schmidt-Schauß, M. Marchiori, and S. Panitz. Modular termination of $r$-consistent and left-linear term rewriting systems. Theoretical Computer Science, 149(2):361-374, 1995.

[23] Y. Toyama. Counterexamples to termination for the direct sum of term rewriting systems. Information Processing Letters, 25:141-143, 1987.

[24] Y. Toyama. On the Church-Rosser property for the direct sum of term rewriting systems. Journal of the ACM, 34(1):128-143, 1987.

[25] Y. Toyama, J. Klop, and H. Barendregt. Termination for direct sums of left-linear complete term rewriting systems. Journal of the ACM, 42(6):1275-1304, 1995.

[26] H. Zantema. Termination of term rewriting: interpretation and type elimination. Journal of Symbolic Computation, 17:23-50, 1994. 


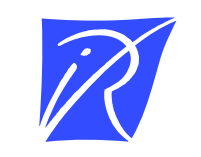

Unit'e de recherche INRIA Lorraine, Technop^ole de Nancy-Brabois, Campus scientifique, 615 rue du Jardin Botanique, BP 101, 54600 VILLERS LÈS NANCY

Unit'e de recherche INRIA Rennes, Irisa, Campus universitaire de Beaulieu, 35042 RENNES Cedex Unit’e de recherche INRIA Rh^one-Alpes, 655, avenue de l'Europe, 38330 MONTBONNOT ST MARTIN Unit'e de recherche INRIA Rocquencourt, Domaine de Voluceau, Rocquencourt, BP 105, 78153 LE CHESNAY Cedex

Unit'e de recherche INRIA Sophia-Antipolis, 2004 route des Lucioles, BP 93, 06902 SOPHIA-ANTIPOLIS Cedex

Éditeur

INRIA, Domaine de Voluceau, Rocquencourt, BP 105, 78153 LE CHESNAY Cedex (France)

ISSN 0249-6399 